\title{
ON THE CLASSIFICATION OF SCHREIER EXTENSIONS OF MONOIDS WITH NON-ABELIAN KERNEL
}

\author{
NELSON MARTINS-FERREIRA, ANDREA MONTOLI, ALEX PATCHKORIA, \\ AND MANUELA SOBRAL
}

\begin{abstract}
We show that any regular (right) Schreier extension of a monoid $M$ by a monoid $A$ induces an abstract kernel $\Phi: M \rightarrow \frac{E n d(A)}{\operatorname{Inn}(A)}$. If an abstract kernel factors through $\frac{S \operatorname{End}(A)}{\operatorname{Inn}(A)}$, where $\operatorname{SEnd}(A)$ is the monoid of surjective endomorphisms of $A$, then we associate to it an obstruction, which is an element of the third cohomology group of $M$ with coefficients in the abelian group $U(Z(A))$ of invertible elements of the center $Z(A)$ of $A$, on which $M$ acts via $\Phi$. An abstract kernel $\Phi: M \rightarrow \frac{\operatorname{SEnd}(A)}{\operatorname{Inn}(A)}\left(\operatorname{resp} . \Phi: M \rightarrow \frac{A u t(A)}{\operatorname{Inn}(A)}\right)$ is induced by a regular weakly homogeneous (resp. homogeneous) Schreier extension of $M$ by $A$ if and only if its obstruction is zero. We also show that the set of isomorphism classes of regular weakly homogeneous (resp. homogeneous) Schreier extensions inducing a given abstract kernel $\Phi: M \rightarrow \frac{\operatorname{SEnd}(A)}{\operatorname{Inn}(A)}$ (resp. $\left.\Phi: M \rightarrow \frac{\operatorname{Aut}(A)}{\operatorname{Inn}(A)}\right)$, when it is not empty, is in bijection with the second cohomology group of $M$ with coefficients in $U(Z(A))$.
\end{abstract}

\section{INTRODUCTION}

The classification of extensions is a classical problem in group theory. It is well known that extensions with abelian kernel inducing the same action are classified by the 2-dimensional cohomology group. The case of non-abelian kernels was studied by Schreier $[25,26]$ : to any group extension

$$
0 \longrightarrow A \longrightarrow B \longrightarrow G \longrightarrow 1
$$

he associated a group homomorphism $\Phi: G \rightarrow \frac{A u t(A)}{\operatorname{Inn}(A)}$, called abstract kernel of the extension, and he determined conditions on such a homomorphism $\Phi$ in order to get the existence of extensions having it as abstract kernel. (The notation for group extensions is borrowed from Mac Lane's book [16], and it is justified by the fact that we will use the multiplicative notation for the group $G$ and the additive one for the other groups.) Later, Eilenberg and Mac Lane [10] gave an interpretation of such results in terms of cohomology: to an abstract kernel $\Phi$ can be associated an element $\operatorname{Obs}(\Phi)$, called obstruction of the abstract kernel, of the third cohomology group $H^{3}(G, Z(A))$, where $Z(A)$ is the center of $A$ and the left $G$-module structure on $Z(A)$ is induced by $\Phi$. Then $\Phi$ is induced by an extension if and only if $\operatorname{Obs}(\Phi)$ is the zero element of $H^{3}(G, Z(A))$. Moreover, if there is an extension inducing $\Phi$, then the set of isomorphism classes of the extensions inducing it is in bijection with the second cohomology group $H^{2}(G, Z(A))$. See, for example, $[10,16]$ for a detailed account of this result.

Date: November 4, 2019.

2010 Mathematics Subject Classification. 20M32, 20M50, 20J99, 18 G50.

Key words and phrases. monoid, Schreier extension, obstruction, Eilenberg-Mac Lane cohomology of monoids. 
The same kind of result was then extended to other algebraic structures, such as associative algebras [12] and Lie algebras [13] over a field, rings [15], categories of interest [19], categorical groups [11, 7]. A categorical approach to this problem was initiated by Bourn in [1] and then generalized in $[6,2,9,8]$ to the context of semi-abelian [14] action accessible [3] categories.

The situation for monoid extensions is more complicated. Schreier extensions of monoids, a direct generalization of group extensions, were introduced by Rédei [24]. In [27] the Schreier extensions of a monoid $M$ by an $M$-module $A$ were classified by $H^{2}(M, A)$, the classical second cohomology group of $M$ with coefficients in the $M$-module $A$. Then, in $[20,22]$ the Schreier extensions of a monoid $M$ by an $M$-semimodule $A$ (i.e. a commutative monoid on which $M$ acts) have been classified by means of the second cohomology monoid $H^{2}(M, A)$, of a cohomology theory of monoids with coefficients in semimodules [21, 22] which generalizes the classical Eilenberg-Mac Lane cohomology of monoids. The problem of classifying Schreier extensions of monoids whose kernels are (not necessarily abelian) groups was studied in [27]. There the abstract kernel is involved in the definition of the extension, because the author of [27] was not able to induce an abstract kernel, i.e. a monoid homomorphism $\Phi: M \rightarrow \frac{\operatorname{End}(A)}{\operatorname{Inn}(A)}$, from a given Schreier extension $0 \longrightarrow A>B \longrightarrow M \longrightarrow 1$.

In the present paper, we show how to induce an abstract kernel from a regular (see Definition 3.7) Schreier extension of monoids, a particular case of which is a Schreier extension of a monoid whose kernel is a group. More specifically, in Section 3 we associate to any regular Schreier extension of a monoid $M$ by a monoid $A$ a monoid homomorphism $\Phi: M \rightarrow \frac{\operatorname{End}(A)}{\operatorname{Inn}(A)}$, and in Section 4 we show that there is a canonical representative of such a monoid extension, called the crossed product extension. In Section 5 we show that, if the abstract kernel $\Phi$ takes values in $\frac{\operatorname{SEnd}(A)}{\operatorname{Inn}(A)}$, where $S \operatorname{End}(A)$ is the monoid of surjective endomorphisms of $A$, then it is possible to associate to $\Phi$ an element $\operatorname{Obs}(\Phi)$ of the third cohomology group $H^{3}(M, U(Z(A)))$, where $U(Z(A))$ is the abelian group of invertible elements of the center $Z(A)$ of $A$, and the action of $M$ on $U(Z(A))$ is induced by $\Phi$. Moreover, we show that an abstract kernel $\Phi$ is induced by an extension if and only if $\operatorname{Obs}(\Phi)$ is the zero element of the third cohomology group. Finally, in Section 6 we show that the set $\operatorname{Ext}(M, A, \Phi)$ of isomorphism classes of regular weakly homogeneous (resp. homogeneous) Schreier extensions of $M$ by $A$ (see Definition 3.11) which induce the same abstract kernel $\Phi: M \rightarrow \frac{\operatorname{SEnd}(A)}{\operatorname{Inn}(A)}$ (resp. $\Phi: M \rightarrow \frac{\operatorname{Aut}(A)}{\operatorname{Inn}(A)}$ ), when it is not empty, is in bijection with the second cohomology group $H^{2}(M, U(Z(A)))$ of $M$ with coefficients in the $M$-module $U(Z(A))$. This is done, as for the classical case of extensions of groups, by showing that there is a simply transitive action of the abelian group $H^{2}(M, U(Z(A)))$ on the set $\operatorname{Ext}(M, A, \Phi)$. Hence our approach is very similar to the classical one for groups, yielding a new, additional interpretation of the classical Eilenberg-Mac Lane cohomology in terms of monoid extensions.

\section{Preliminaries}

In this section we recall some notions we need in the rest of the paper and we fix some notations.

Given a monoid $M$, we will denote by $Z(M)$ the center of $M$, namely

$$
Z(M)=\{z \in M \mid z m=m z \quad \text { for all } m \in M\},
$$


and by $U(M)$ the group of invertible elements of $M$.

Definition 2.1. Given a monoid $M$ and a subgroup $H$ (i.e. a subgroup $H$ of the group $U(M)$ ), we say that $H$ is

- right normal if, for all $m \in M, m H \subseteq H m$, where

$$
m H=\{m h \mid h \in H\}, \quad H m=\{h m \mid h \in H\} ;
$$

- left normal if, for all $m \in M, H m \subseteq m H$;

- normal if it is both right and left normal, i.e., $m H=H m$.

Note that $H$ is right normal in $M$ if and only if $H$ is left normal in $M^{o p}$.

If $H$ is a subgroup of a monoid $M$, the relation on $M$ defined by

$$
m_{1} \sim m_{2} \quad \Leftrightarrow \quad m_{1}=h m_{2} \quad \text { for some } h \in H
$$

is an equivalence relation on $M$, called the right coset relation. The equivalence class of an element $m$ is $c l(m)=H m$. We will denote by $\frac{M}{H}$ the quotient set. Similarly we can define the left coset relation.

Proposition 2.2. If $H$ is right normal in $M$, then the operation

$$
H m_{1} \cdot H m_{2}=H m_{1} m_{2}
$$

is well defined, and $\left(\frac{M}{H}, \cdot, H\right)$ is a monoid.

Proof. If $H m_{1}=H m_{1}^{\prime}$ and $H m_{2}=H m_{2}^{\prime}$, then there exist $h_{1}, h_{2} \in H$ such that

$$
m_{1}=h_{1} m_{1}^{\prime}, \quad m_{2}=h_{2} m_{2}^{\prime} .
$$

Hence $m_{1} m_{2}=h_{1} m_{1}^{\prime} h_{2} m_{2}^{\prime}$. Since $H$ is right normal, there exists $h_{3} \in H$ such that $m_{1}^{\prime} h_{2}=h_{3} m_{1}^{\prime}$, and so

$$
m_{1} m_{2}=h_{1} m_{1}^{\prime} h_{2} m_{2}^{\prime}=h_{1} h_{3} m_{1}^{\prime} m_{2}^{\prime},
$$

which proves that $H m_{1} m_{2}=H m_{1}^{\prime} m_{2}^{\prime}$.

The same happens for the left coset relation, when $H$ is left normal.

Example 2.3. If $A$ is a monoid, $\operatorname{End}(A)$ is the monoid of endomorphisms of $A$ (w.r.t. the usual composition of functions, $(g f)(a)=g(f(a)))$, and $\operatorname{Inn}(A)$ is the subgroup of inner automorphisms induced by the invertible elements of $A$, then $\operatorname{Inn}(A)$ is right normal, but not left normal, in $\operatorname{End}(A)$. Indeed, if $\varphi \in \operatorname{End}(A)$, $\mu_{g} \in \operatorname{Inn}(A)$, then

$$
\left(\varphi \mu_{g}\right)(a)=\varphi\left(\mu_{g}(a)\right)=\varphi\left(g a g^{-1}\right)=\varphi(g) \varphi(a) \varphi(g)^{-1}=\mu_{\varphi(g)}(\varphi(a))=\left(\mu_{\varphi(g)} \varphi\right)(a),
$$

hence $\varphi \mu_{g}=\mu_{\varphi(g)} \varphi$, which shows that $\operatorname{Inn}(A)$ is right normal in $\operatorname{End}(A)$. But it is not left normal, in general. A concrete counterexample is the following. If $A$ is the symmetric group $S_{3}$, consider the endomorphism $f$ of $S_{3}$ defined by

$$
f(i d)=f((123))=f((132))=i d, \quad f((12))=f((13))=f((23))=(12) .
$$

Then, for every element $s \in S_{3}, f \mu_{s}=f$, and so $f \operatorname{Inn}(A)=\{f\}$, but the endomorphism $\mu_{(13)} f$ is different from $f$, indeed:

$$
\mu_{(13)} f((12))=\mu_{(13)} f((13))=\mu_{(13)} f((23))=(13)(12)(13)^{-1}=(23),
$$

and so $\operatorname{Inn}(A) f$ is not contained in $f \operatorname{Inn}(A)$.

Proposition 2.4. If $G$ is a group, then $\operatorname{Inn}(G)$ is normal in the monoid Epi $(G)$ of epimorphisms of $G$. 
Proof. As we observed before, $\operatorname{Inn}(G)$ is right normal in $\operatorname{End}(G)$, and so it is right normal in $\operatorname{Epi}(G)$, too. Let us prove that it is also left normal. If $\varphi \in \operatorname{Epi}(G)$ and $g \in G$, let $g^{\prime} \in G$ be such that $\varphi\left(g^{\prime}\right)=g$ (since $G$ is a group, $\varphi$ is surjective). Then, for all $x \in G$, we have

$$
\left(\mu_{g} \varphi\right)(x)=g \varphi(x) g^{-1}=\varphi\left(g^{\prime}\right) \varphi(x) \varphi\left(g^{\prime}\right)^{-1}=\varphi\left(g^{\prime} x g^{\prime-1}\right)=\left(\varphi \mu_{g^{\prime}}\right)(x),
$$

hence $\varphi \operatorname{Inn}(G)=\operatorname{Inn}(G) \varphi$.

\section{SCHREIER EXTENSIONS}

Definition 3.1 ([24]). Let

$$
E: 0 \longrightarrow A>\stackrel{\kappa}{\longrightarrow} B \stackrel{\sigma}{\longrightarrow} M \longrightarrow 1
$$

be a sequence of monoids and monoid homomorphisms such that $\sigma$ is a surjection, $\kappa$ is an injection and $\kappa(A)=\{b \in B \mid \sigma(b)=1\}$ (i.e. $\kappa$ is the kernel of $\sigma$ ). $E$ is a (right) Schreier extension of $M$ by $A$ (some authors would say " $A$ by $M$ ") if, for every $x \in M$, there exists an element $u_{x} \in \sigma^{-1}(x)$ such that for every $b \in \sigma^{-1}(x)$ there exists a unique $a \in A$ such that

$$
b=\kappa(a)+u_{x} .
$$

The elements $u_{x}$, for $x \in M$, will be called the representatives of $E$. We will always choose $u_{1}=0$ (we use the multiplicative notation for $M$ and the additive one for the other monoids involved).

Note that, if (1) is a Schreier extension, then $\sigma$ is the cokernel of $\kappa$. Indeed, suppose that $f: B \rightarrow C$ is a monoid homomorphism such that $f \kappa(a)=0$ for all $a \in A$. Define a map $g: M \rightarrow C$ by putting $g(x)=f(b), b \in \sigma^{-1}(x)$. If $\sigma\left(b_{1}\right)=$ $x=\sigma\left(b_{2}\right)$, then $b_{1}=\kappa\left(a_{1}\right)+u_{x}$ and $b_{2}=\kappa\left(a_{2}\right)+u_{x}$, whence $f\left(b_{1}\right)=f\left(u_{x}\right)=f\left(b_{2}\right)$. Hence $g$ is well defined. Clearly, $g$ is a monoid homomorphism and $g \sigma=f$. The uniqueness of such a homomorphism $g$ is also clear.

Example 3.2. Let $\mathbb{N}$ be the commutative monoid of natural numbers, with the usual sum, and let $C_{m}(t)$ denote the multiplicative cyclic group of order $m$ with generator $t$. The sequence

$$
0 \longrightarrow \mathbb{N}>\bar{m} \longrightarrow \mathbb{N} \stackrel{p}{\longrightarrow} C_{m}(t) \longrightarrow 1,
$$

where $\bar{m}(1)=m$ and $p(1)=t$, is a Schreier extension of $C_{m}(t)$ by $\mathbb{N}$, with representatives given by $0,1, \ldots, m-1$.

From now on, we will treat $\kappa$ just as an inclusion.

Proposition 3.3. Let $E$ be a Schreier extension as in (1), with representatives $u_{x}$, $x \in M$. An element $b \in \sigma^{-1}(x)$ is another representative of $x$ for $E$ if and only if $b=g+u_{x}$ for some $g \in U(A)$.

Proof. Since $u_{x}$ is a representative, there exists a unique $a \in A$ such that $b=a+u_{x}$. Moreover, if $b$ is a representative for $E$, then there is a unique $a^{\prime} \in A$ such that $u_{x}=a^{\prime}+b$. Hence we get

$$
b=a+a^{\prime}+b .
$$

By the uniqueness in the Schreier condition, we get $a+a^{\prime}=0$. Similarly, from the equality

$$
u_{x}=a^{\prime}+a+u_{x},
$$

we get $a^{\prime}+a=0$, and so $a$ is invertible. Conversely, if $b=g+u_{x}$ with $g \in U(A)$, then for every $b^{\prime} \in \sigma^{-1}(x)$ there exists a unique $a^{\prime} \in A$ such that

$$
b^{\prime}=a^{\prime}+u_{x}=a^{\prime}-g+b .
$$


Moreover, if $a_{1}+b=a_{2}+b$, then $a_{1}+g+u_{x}=a_{2}+g+u_{x}$, then the uniqueness in the Schreier condition implies $a_{1}+g=a_{2}+g$, and hence $a_{1}=a_{2}$, because $g$ is invertible.

Lemma 3.4. Let $E$ be a Schreier extension as in (1), with representatives $u_{x}$, $x \in M$. For $a \in A$, let $a^{\prime}$ be the unique element in $A$ such that $u_{x}+a=a^{\prime}+u_{x}$. If $a \in U(A)$, then $a^{\prime} \in U(A)$, too.

Proof. There exists a unique $a^{\prime \prime} \in A$ such that $u_{x}+(-a)=a^{\prime \prime}+u_{x}$. From the equality $u_{x}+a=a^{\prime}+u_{x}$ we obtain

$$
u_{x}=a^{\prime}+u_{x}-a=a^{\prime}+a^{\prime \prime}+u_{x},
$$

and the uniqueness in the Schreier condition implies $a^{\prime}+a^{\prime \prime}=0$. Similarly, from the equality $u_{x}+(-a)=a^{\prime \prime}+u_{x}$ we get

$$
u_{x}=a^{\prime \prime}+u_{x}+a=a^{\prime \prime}+a^{\prime}+u_{x},
$$

from where we obtain $a^{\prime \prime}+a^{\prime}=0$.

Proposition 3.5. Let $E$ be a Schreier extension as in (1), and let $u_{x}, u_{y}, v_{x}, v_{y}$ be representatives, for $x, y \in M$. If $u_{x}+u_{y}$ is a representative, then so is $v_{x}+v_{y}$.

Proof. Thanks to Proposition 3.3, we know that there exist $g_{1}, g_{2} \in U(A)$ such that

$$
v_{x}=g_{1}+u_{x}, \quad v_{y}=g_{2}+u_{y} .
$$

Moreover, there exists a unique $h \in A$ such that $u_{x}+g_{2}=h+u_{x}$, and such an $h$ is invertible thanks to the previous lemma. Then we have

$$
v_{x}+v_{y}=g_{1}+u_{x}+g_{2}+u_{y}=g_{1}+h+u_{x}+u_{y},
$$

with $g_{1}+h \in U(A)$. Then the thesis follows from Proposition 3.3.

Let $E$ be a Schreier extension as in (1), with representatives $u_{x}, x \in M$. We already observed that, for all $a \in A$, there is a unique element $a^{\prime} \in A$ such that $u_{x}+a=a^{\prime}+u_{x}$. This defines a map $\varphi(x): A \rightarrow A$ sending $a$ to $a^{\prime}$.

Proposition 3.6. $\quad$ (a) For every $x \in M$ we have that $\varphi(x) \in \operatorname{End}(A)$;

(b) if $v_{x}$ is another representative, and $\psi(x): A \rightarrow A$ is the induced endomorphism of $A$, then $\psi(x)=\mu_{g} \varphi(x)$ with $g \in U(A)$.

Proof. (a) From the obvious equality $u_{x}+0=0+u_{x}$, we get $\varphi(x)(0)=0$. Moreover, on one hand

$$
u_{x}+a_{1}+a_{2}=\varphi(x)\left(a_{1}+a_{2}\right)+u_{x},
$$

while, on the other hand

$$
u_{x}+a_{1}+a_{2}=\varphi(x)\left(a_{1}\right)+u_{x}+a_{2}=\varphi(x)\left(a_{1}\right)+\varphi(x)\left(a_{2}\right)+u_{x} .
$$

By the uniqueness we get that $\varphi(x)\left(a_{1}+a_{2}\right)=\varphi(x)\left(a_{1}\right)+\varphi(x)\left(a_{2}\right)$.

(b) From Proposition 3.3 we know that $v_{x}=g+u_{x}$ with $g \in U(A)$. Moreover, for all $a \in A$,

Therefore

$$
v_{x}+a=\psi(x)(a)+v_{x}
$$

$$
v_{x}+a=g+u_{x}+a=g+\varphi(x)(a)+u_{x}=g+\varphi(x)(a)-g+v_{x} .
$$

This means that

$$
\psi(x)(a)=g+\varphi(x)(a)-g=\left(\mu_{g} \varphi(x)\right)(a)
$$

for all $a \in A$. 
The previous proposition implies that, for a Schreier extension $E$ as in (1), there is an induced well-defined map

$$
\Phi: M \rightarrow \frac{\operatorname{End}(A)}{\operatorname{Inn}(A)},
$$

given by $\Phi(x)=c l\left(\varphi(x)\right.$ ), such that $\Phi(1)=c l\left(i d_{A}\right)$ (see Proposition 2.2 and Example 2.3). In order to have that $\Phi$ is a monoid homomorphism, we need an additional assumption:

Definition 3.7. Let $E$ be a Schreier extension as in (1). We say that $E$ is a regular Schreier extension if, whenever $u_{x}$ and $u_{y}$ are representatives for $E$, then so is $u_{x}+u_{y}$ (such extensions are called normal Schreier extensions in [22]).

Proposition 3.8. If $E$ is a regular Schreier extension, then the map (2) is a monoid homomorphism.

Proof. Let $x, y \in M$, and let $u_{x}, u_{y}$ and $u_{x y}$ be representatives. We have the corresponding $\varphi(x), \varphi(y), \varphi(x y) \in \operatorname{End}(A)$ with

$$
u_{x}+a=\varphi(x)(a)+u_{x}, \quad u_{y}+a=\varphi(y)(a)+u_{y}, \quad u_{x y}+a=\varphi(x y)(a)+u_{x y}
$$

for all $a \in A$. Since $E$ is regular, $u_{x}+u_{y}$ is a representative, hence $u_{x}+u_{y}=g+u_{x y}$ for some $g \in U(A)$. On one hand we have

$$
u_{x}+u_{y}+a=g+u_{x y}+a=g+\varphi(x y)(a)+u_{x y},
$$

while on the other hand

$u_{x}+u_{y}+a=u_{x}+\varphi(y)(a)+u_{y}=\varphi(x)(\varphi(y)(a))+u_{x}+u_{y}=\varphi(x)(\varphi(y)(a))+g+u_{x y}$.

Therefore

$$
g+\varphi(x y)(a)=\varphi(x)(\varphi(y)(a))+g
$$

whence

$$
\varphi(x)(\varphi(y)(a))=g+\varphi(x y)(a)-g .
$$

This means that $\varphi(x) \varphi(y)=\mu_{g} \varphi(x y)$, i.e. $\Phi(x) \Phi(y)=\Phi(x y)$.

Definition 3.9. Given a regular Schreier extension $E$ as in (1), the induced monoid homomorphism

$$
\Phi: M \rightarrow \frac{\operatorname{End}(A)}{\operatorname{Inn}(A)}
$$

is called the abstract kernel induced by the extension E. More generally, we will call abstract kernel any such homomorphism, even when it is not induced by an extension.

The following proposition gives examples of regular Schreier extensions:

Proposition 3.10. Let $E$ be a Schreier extension as in (1) such that $A$ is a group (such extensions are called special Schreier extensions in $[4,5,17,18])$. Then every element of $B$ is a representative and therefore $E$ is regular.

Proof. Let $x \in M$, and let $u_{x}$ be a representative. For every $b \in \sigma^{-1}(x)$ there exists (a unique) $a \in A$ such that $b=a+u_{x}$. Being $A$ a group, $a$ is invertible. Then it follows from Proposition 3.3 that $b$ is a representative. Thus, every element of $B$ is a representative, and hence $E$ is regular.

Note that the extension of Example 3.2 serves as an example of Schreier extension which is not regular.

Definition 3.11. A Schreier extension $E$ as in (1), with representatives $u_{x}, x \in M$, is: 
(a) weakly homogeneous if for all $b \in \sigma^{-1}(x)$ there exists $a \in A$ such that $b=u_{x}+a$

(b) homogeneous if for all $b \in \sigma^{-1}(x)$ there is a unique such $a$.

Note that, thanks to Proposition 3.3, this definition does not depend on the choice of representatives. (Indeed, for any representative $v_{x}$, we have $u_{x}=g+$ $v_{x}, g \in U(A)$. If (a) holds, then $b=-g+g+b=-g+u_{x}+a^{\prime}=v_{x}+a^{\prime}$. If (b) holds, then we have $v_{x}+a_{1}=v_{x}+a_{2} \quad \Rightarrow g+v_{x}+a_{1}=g+v_{x}+a_{2} \quad \Rightarrow$ $u_{x}+a_{1}=u_{x}+a_{2} \quad \Rightarrow \quad a_{1}=a_{2}$.)

The following proposition is a generalization of Proposition 3.8 in [5], where only split extensions were considered:

Proposition 3.12. Let $E$ be a Schreier extension as in (1), with representatives $u_{x}, x \in M$. Let $\varphi(x): A \rightarrow A$ be the induced endomorphism of $A$ relative to the element $x \in M$. Then:

(a) $E$ is weakly homogeneous if and only if $\varphi(x)$ is surjective for all $x \in M$;

(b) $E$ is homogeneous if and only if $\varphi(x) \in \operatorname{Aut}(A)$ for all $x \in M$.

Proof. (a) Suppose that $E$ is weakly homogeneous, and consider $x \in M$. Given $a \in A$, there exists $a^{\prime} \in A$ such that

$$
a+u_{x}=u_{x}+a^{\prime},
$$

from which we obtain that $\varphi(x)\left(a^{\prime}\right)=a$, and so $\varphi(x)$ is surjective. Conversely, suppose that $\varphi(x)$ is surjective. Given $b \in \sigma^{-1}(x)$, there exists a unique $a \in A$ such that $b=a+u_{x}$ (because $E$ is Schreier). The surjectivity of $\varphi(x)$ implies the existence of $a^{\prime} \in A$ such that $\varphi(x)\left(a^{\prime}\right)=a$. Hence

$$
u_{x}+a^{\prime}=\varphi(x)\left(a^{\prime}\right)+u_{x}=a+u_{x}=b .
$$

(b) Suppose that $E$ is homogeneous. We already know that, for all $x \in M$, $\varphi(x)$ is surjective. Suppose that $\varphi(x)\left(a_{1}\right)=\varphi(x)\left(a_{2}\right)$. Then

$$
u_{x}+a_{1}=\varphi(x)\left(a_{1}\right)+u_{x}=\varphi(x)\left(a_{2}\right)+u_{x}=u_{x}+a_{2},
$$

and the uniqueness in the definition of a homogeneous Schreier extension implies that $a_{1}=a_{2}$, and so $\varphi(x)$ is injective. Conversely, suppose that $\varphi(x) \in \operatorname{Aut}(A)$. We already know that $E$ is weakly homogeneous. If $u_{x}+a_{1}=u_{x}+a_{2}$, then

$$
\varphi(x)\left(a_{1}\right)+u_{x}=\varphi(x)\left(a_{2}\right)+u_{x} .
$$

Being $E$ Schreier, this implies that $\varphi(x)\left(a_{1}\right)=\varphi(x)\left(a_{2}\right)$, and the injectivity of $\varphi(x)$ gives us that $a_{1}=a_{2}$.

From now on, $S E n d(A)$ denotes the monoid of surjective endomorphisms of a monoid $A$.

The previous proposition shows that a regular weakly homogeneous Schreier extension $E$ as in (1) induces a monoid homomorphism $\Phi: M \rightarrow \frac{\operatorname{SEnd}(A)}{\operatorname{Inn}(A)}$, while a regular homogeneous Schreier extension induces a monoid homomorphism $\Phi: M \rightarrow \frac{\operatorname{Aut}(A)}{\operatorname{Inn}(A)}$.

The following result is a generalization of Proposition 3.4 in [5]:

Proposition 3.13. If $E: 0 \longrightarrow A>\stackrel{\kappa}{\longrightarrow} B \stackrel{\sigma}{\longrightarrow} M \longrightarrow 1$ is a regular Schreier extension and $M$ is a group, then $E$ is homogeneous. 
Proof. Given representatives $u_{x}, x \in M$, with $u_{1}=0$, consider the induced endomorphisms $\varphi(x): A \rightarrow A$. If $\varphi(x)\left(a_{1}\right)=\varphi(x)\left(a_{2}\right)$, then $u_{x}+a_{1}=u_{x}+a_{2}$, whence

$$
u_{x^{-1}}+u_{x}+a_{1}=u_{x^{-1}}+u_{x}+a_{2} .
$$

Since $E$ is regular, $u_{x^{-1}}+u_{x}$ is a representative of 1 , hence it is an invertible element of $A$ (by Proposition 3.3). This implies that $a_{1}=a_{2}$, and thus $\varphi(x)$ is injective. Moreover, since $E$ is regular, we have

$$
\varphi(x) \varphi\left(x^{-1}\right)=\mu_{g} \varphi\left(x x^{-1}\right)=\mu_{g} \varphi(1)=\mu_{g}
$$

for some $g \in U(A)$ (see Proposition 3.8). Being $\mu_{g}$ an automorphism, we deduce that $\varphi(x)$ is surjective. Then the thesis follows from Proposition 3.12.

Example 3.14. Consider the sequence

$$
A \stackrel{\kappa}{\longrightarrow} A \times{ }_{g} C_{2}(t) \stackrel{\sigma}{\longrightarrow} C_{2}(t),
$$

where $A$ is any monoid, $C_{2}(t)$ is the cyclic group of order 2 with generator $t, g$ is a fixed element of $U(Z(A)), C_{2}(t)$ acts on the monoid $A$ in a way that $t \cdot g=g$, and $A \times{ }_{g} C_{2}(t)$ is the cartesian product $A \times C_{2}(t)$ with the monoid operation defined by

$$
\begin{gathered}
\left(a_{1}, 1\right)+\left(a_{2}, 1\right)=\left(a_{1}+a_{2}, 1\right), \quad\left(a_{1}, 1\right)+\left(a_{2}, t\right)=\left(a_{1}+a_{2}, t\right), \\
\left(a_{1}, t\right)+\left(a_{2}, 1\right)=\left(a_{1}+t \cdot a_{2}, t\right), \quad\left(a_{1}, t\right)+\left(a_{2}, t\right)=\left(a_{1}+t \cdot a_{2}+g, 1\right) .
\end{gathered}
$$

It is straightforward to check that this operation is associative and that $(0,1)$ is the neutral element. The morphism $\sigma$ is just the canonical projection, while $\kappa(a)=$ $(a, 1)$. This sequence is a regular homogeneous Schreier extension. In order to show that it is Schreier, it suffices to choose the representatives $u_{1}=(0,1)$ and $u_{t}=(0, t)$. Thanks to Proposition 3.5, regularity is proved just by observing that the element

$$
u_{t}+u_{t}=(0, t)+(0, t)=(g, 1)=(g, 1)+(0,1)=(g, 1)+u_{1}
$$

is a representative, since $(g, 1)$ is invertible (see Proposition 3.3). The previous proposition implies that the extension is homogeneous.

Several other examples of Schreier and homogeneous Schreier extensions may be found in $[4,5]$.

\section{THE CROSSED PRODUCT EXTENSION}

Let $E: 0 \longrightarrow A>\stackrel{\kappa}{\longrightarrow} B \stackrel{\sigma}{\longrightarrow} M \longrightarrow 1$ be a regular Schreier extension, with representatives $u_{x}, x \in M$. Being $E$ regular, we know that for all $x, y \in M$ the element $u_{x}+u_{y}$ is a representative. Thanks to Proposition 3.3 we get that there exists a unique element $f(x, y) \in U(A)$ such that $u_{x}+u_{y}=f(x, y)+u_{x y}$. This defines a map

such that

$$
f: M \times M \rightarrow U(A)
$$

$$
f(x, 1)=f(1, y)=0
$$

for all $x, y \in M$ (because we are assuming that $u_{1}=0$ ). Then we have, on one hand

and, on the other hand

$$
u_{x}+u_{y}+u_{z}=f(x, y)+u_{x y}+u_{z}=f(x, y)+f(x y, z)+u_{x y z},
$$

$u_{x}+u_{y}+u_{z}=u_{x}+f(y, z)+u_{y z}=\varphi(x)(f(y, z))+u_{x}+u_{y z}=\varphi(x)(f(y, z))+f(x, y z)+u_{x y z}$, where $\varphi: M \rightarrow \operatorname{End}(A)$ is the map defined by the following equality (as we explained in the previous section):

$$
u_{x}+a=\varphi(x)(a)+u_{x} .
$$


Whence

$$
\varphi(x)(f(y, z))+f(x, y z)=f(x, y)+f(x y, z) \quad \text { for all } x, y, z \in M .
$$

Furthermore, for every $x, y \in M$ and every $a \in A$ we have, on one hand $u_{x}+u_{y}+a=u_{x}+\varphi(y)(a)+u_{y}=\varphi(x) \varphi(y)(a)+u_{x}+u_{y}=\varphi(x) \varphi(y)(a)+f(x, y)+u_{x y}$, and, on the other hand

$$
u_{x}+u_{y}+a=f(x, y)+u_{x y}+a=f(x, y)+\varphi(x y)(a)+u_{x y},
$$

whence

$$
\varphi(x) \varphi(y)(a)+f(x, y)=f(x, y)+\varphi(x y)(a)
$$

Being $f(x, y)$ invertible, the last equality implies that

$$
\varphi(x) \varphi(y)(a)=f(x, y)+\varphi(x y)(a)-f(x, y) .
$$

Thus

$$
\varphi(x) \varphi(y)=\mu_{f(x, y)} \varphi(x y) \quad \text { for all } x, y \in M .
$$

Proposition 4.1. Let monoids $M, A$ and maps $\varphi: M \rightarrow \operatorname{End}(A), f: M \times M \rightarrow U(A)$ such that, for all $x, y, z \in M$,

$$
\begin{gathered}
\varphi(1)=i d_{A}, \quad f(x, 1)=f(1, y)=0, \quad \varphi(x) \varphi(y)=\mu_{f(x, y)} \varphi(x y), \\
\varphi(x)(f(y, z))+f(x, y z)=f(x, y)+f(x y, z),
\end{gathered}
$$

be given. Then the set $[A, \varphi, f, M]$ of all pairs $(a, x) \in A \times M$ with the operation defined by

$$
\left(a_{1}, x\right)+\left(a_{2}, y\right)=\left(a_{1}+\varphi(x)\left(a_{2}\right)+f(x, y), x y\right)
$$

is a monoid, and the sequence

$$
A \stackrel{i}{\longrightarrow}[A, \varphi, f, M] \stackrel{p}{\longrightarrow} M, \quad i(a)=(a, 1), \quad p(a, x)=x,
$$

is a regular Schreier extension of $M$ by $A$, called the crossed product extension, such that the induced monoid homomorphism $\Phi: M \rightarrow \frac{\operatorname{End}(A)}{\operatorname{Inn}(A)}$ sends $x \in M$ to the equivalence class of $\varphi(x)$. Furthermore, a pair $(a, x)$ is a representative if and only if $a \in U(A)$.

Proof. It is straightforward to show that the operation is associative and that $(0,1)$ is its neutral element. The maps $i$ and $p$ are clearly monoid homomorphisms, $p$ is surjective and $i$ is injective, and the image of $i$ is the kernel of $p$. Let us show that we get a regular Schreier extension. For any $x \in M$, we consider the element $u_{x}=(0, x)$. These elements are representatives: indeed, every element $(a, x) \in A \times M$ can be written as

$$
(a, x)=(a, 1)+(0, x),
$$

and such writing is unique, because the equality

$$
\left(a_{1}, 1\right)+(0, x)=\left(a_{2}, 1\right)+(0, x)
$$

implies $\left(a_{1}, x\right)=\left(a_{2}, x\right)$, and hence $a_{1}=a_{2}$. So, the extension is Schreier. Proposition 3.3, together with the equality $(a, x)=(a, 1)+(0, x)$, implies that $(a, x)$ is a representative if and only if $a \in U(A)$. It remains to show that the extension is regular. For all $x, y \in M$, we have

$$
\begin{gathered}
u_{x}+u_{y}=(0, x)+(0, y)=(0+\varphi(x)(0)+f(x, y), x y)=(f(x, y), x y)= \\
=(f(x, y), 1)+(0, x y)=(f(x, y), 1)+u_{x y}
\end{gathered}
$$


and then, since $f(x, y) \in U(A), u_{x}+u_{y}$ is a representative by Proposition 3.3. Hence, thanks to Proposition 3.5, the extension is regular. Furthermore, for all $a \in A$, we have

$u_{x}+i(a)=(0, x)+(a, 1)=(\varphi(x)(a), x)=(\varphi(x)(a), 1)+(0, x)=i(\varphi(x)(a))+u_{x}$, which means that $\Phi$ sends $x \in M$ to the equivalence class of $\varphi(x)$.

Remark 4.2. If, in the previous proposition, we have that $\varphi: M \rightarrow S E n d(A)$, then the crossed product extension is weakly homogeneous. Indeed, every element $(a, x) \in A \times M$ can be written as

$$
(a, x)=(0, x)+\left(a^{\prime}, 1\right),
$$

where $a^{\prime} \in A$ is such that $\varphi(x)\left(a^{\prime}\right)=a$ (such an element exists since $\varphi(x)$ is surjective).

If we have that $\varphi: M \rightarrow A u t(A)$, then the crossed product extension is homogeneous. Indeed, if

$$
(0, x)+\left(a_{1}, 1\right)=(0, x)+\left(a_{2}, 1\right)
$$

then

$$
\left(\varphi(x)\left(a_{1}\right), x\right)=\left(\varphi(x)\left(a_{2}\right), x\right) \quad \Longrightarrow \quad \varphi(x)\left(a_{1}\right)=\varphi(x)\left(a_{2}\right) \quad \Longrightarrow \quad a_{1}=a_{2} .
$$

(See Definition 3.11 and the note after it.)

We recall from [23] the following version of the Short Five Lemma for monoid extensions:

Proposition 4.3 ([23], Proposition 4.5). Consider the following commutative diagram of monoid homomorphisms:

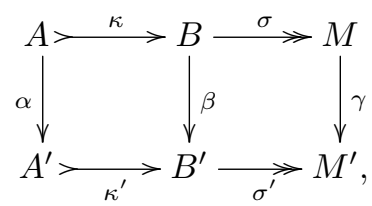

where the two rows are Schreier extensions and the homomorphism $\beta$ sends representatives to representatives. Then:

- if $\alpha$ and $\gamma$ are injective, then $\beta$ also is;

- if $\alpha$ and $\gamma$ are surjective, then $\beta$ also is;

- if $\alpha$ and $\gamma$ are isomorphisms, then $\beta$ is an isomorphism, too.

This fact allows us to prove the following:

Proposition 4.4. Given an abstract kernel $\Phi: M \rightarrow \frac{\operatorname{End}(A)}{\operatorname{Inn}(A)}$, where $A$ and $M$ are monoids, fix an endomorphism $\varphi(x) \in \Phi(x)$ for every element $x \in M$ (with $\left.\varphi(1)=i d_{A}\right)$. Then every regular Schreier extension $E$ as in (1) which induces the abstract kernel $\Phi$ is isomorphic to the crossed product extension

$$
A>\stackrel{i}{\longrightarrow}[A, \varphi, f, M] \stackrel{p}{\longrightarrow} M .
$$

Proof. We take representatives $v_{x}, x \in M$, of $E$, with $v_{1}=0$. Then, for all $a \in A$ and all $x \in M$, we get that

$$
v_{x}+a=\psi(x)(a)+v_{x}
$$

for some $\psi(x) \in \Phi(x)$. Then, for each $x \in M$,

$$
\varphi(x)=\mu_{g(x)} \psi(x)
$$


for some $g(x) \in U(A)$, i.e. for all $a \in A$,

$$
\varphi(x)(a)=g(x)+\psi(x)(a)-g(x) .
$$

We define new representatives by putting $u_{x}=g(x)+v_{x}$, for $x \in M$. Choosing $g(1)=0$, we get $u_{1}=0$. Since $E$ is regular, for all $x, y \in M, u_{x}+u_{y}$ is a representative, hence

$$
u_{x}+u_{y}=f(x, y)+u_{x y}
$$

with $f(x, y) \in U(A)$. Furthermore, for all $a \in A$ and all $x \in M$,

$$
u_{x}+a=g(x)+v_{x}+a=g(x)+\psi(x)(a)+v_{x}=g(x)+\psi(x)(a)-g(x)+u_{x},
$$

that is,

$$
u_{x}+a=\varphi(x)(a)+u_{x} .
$$

Then the maps $\varphi: M \rightarrow \operatorname{End}(A)$ and $f: M \times M \rightarrow U(A)$ satisfy the conditions of Proposition 4.1 (see the considerations before this proposition) and therefore we have the crossed product extension $[A, \varphi, f, M]$. Consider now the diagram

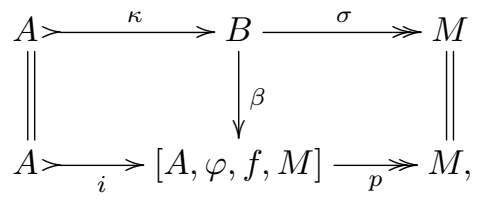

where the map $\beta$ is defined by $\beta(b)=(a, x)$, where $\sigma(b)=x$ and $a$ is the unique element of $A$ such that $b=a+u_{x}$. Then $\beta$ is a monoid homomorphism: clearly $\beta(0)=\beta\left(0+u_{1}\right)=(0,1)$, and moreover

$$
\begin{gathered}
\beta\left(a_{1}+u_{x}+a_{2}+u_{y}\right)=\beta\left(a_{1}+\varphi(x)\left(a_{2}\right)+u_{x}+u_{y}\right)=\beta\left(a_{1}+\varphi(x)\left(a_{2}\right)+f(x, y)+u_{x y}\right)= \\
\left(a_{1}+\varphi(x)\left(a_{2}\right)+f(x, y), x y\right)=\left(a_{1}, x\right)+\left(a_{2}, y\right)=\beta\left(a_{1}+u_{x}\right)+\beta\left(a_{2}+u_{y}\right) .
\end{gathered}
$$

Furthermore

$$
\beta \kappa(a)=\beta\left(a+u_{1}\right)=(a, 1)=i(a)
$$

and

$$
p \beta\left(a+u_{x}\right)=p(a, x)=x=\sigma\left(a+u_{x}\right),
$$

hence the diagram is commutative. Finally, $\beta\left(u_{x}\right)=(0, x)$, and if $w_{x}$ is another representative of $E$ then $w_{x}=g+u_{x}, g \in U(A)$, whence $\beta\left(w_{x}\right)=i(g)+(0, x)$, and so the representatives are preserved by $\beta$ (see Proposition 3.3). Thanks to Proposition 4.3, $\beta$ is an isomorphism.

\section{THE OBSTRUCTION OF AN ABSTRACT KERNEL}

The aim of this section is to show that, to any abstract kernel $\Phi: M \rightarrow \frac{\operatorname{SEnd}(A)}{\operatorname{Inn}(A)}$ (resp. $\left.\Phi: M \rightarrow \frac{\operatorname{Aut}(A)}{\operatorname{Inn}(A)}\right)$, it is possible to associate an element of the third EilenbergMac Lane cohomology group of $M$ with coefficients in the $M$-module $U(Z(A))$, called the obstruction of $\Phi$. Moreover, we will show that the abstract kernel $\Phi: M \rightarrow \frac{\operatorname{SEnd}(A)}{\operatorname{Inn}(A)}$ (resp. $\left.\Phi: M \rightarrow \frac{\operatorname{Aut}(A)}{\operatorname{Inn}(A)}\right)$ is induced by a regular weakly homogeneous (resp. homogeneous) Schreier extension if and only if its obstruction is the zero element of the cohomology group. In order to do this, we first describe how to get from $\Phi$ a structure of $M$-module on $U(Z(A))$.

Proposition 5.1. Given an abstract kernel $\Phi: M \rightarrow \frac{S E n d(A)}{\operatorname{Inn}(A)}$, where $A$ and $M$ are monoids, the center $Z(A)$ of $A$ is an $M$-semimodule w.r.t. the action defined by

$$
x \cdot c=\varphi(x)(c) \quad \text { for } x \in M, c \in Z(A), \varphi(x) \in \Phi(x) .
$$


Proof. We first show that $x \cdot c \in Z(A)$ for all $x \in M, c \in Z(A)$ and $\varphi(x) \in \Phi(x)$. Consider an element $a \in A$. Being $\varphi(x)$ surjective, there exists $a^{\prime} \in A$ such that $\varphi(x)\left(a^{\prime}\right)=a$. Then

$$
\begin{aligned}
& a+\varphi(x)(c)=\varphi(x)\left(a^{\prime}\right)+\varphi(x)(c)=\varphi(x)\left(a^{\prime}+c\right)= \\
& =\varphi(x)\left(c+a^{\prime}\right)=\varphi(x)(c)+\varphi(x)\left(a^{\prime}\right)=\varphi(x)(c)+a .
\end{aligned}
$$

Now, it remains to show that the definition above does not depend on the choice of the representative $\varphi(x)$ of the class $\Phi(x)$ in the quotient $\frac{S E n d(A)}{\operatorname{Inn}(A)}$. To do that, consider another representative $\psi(x) \in S \operatorname{End}(A)$. Then there is an element $g \in U(A)$ such that $\psi(x)=\mu_{g} \varphi(x)$. So, we get

$$
\psi(x)(c)=\mu_{g} \varphi(x)(c)=g+\varphi(x)(c)-g=\varphi(x)(c)+g-g=\varphi(x)(c),
$$

where we are using that $\varphi(x)(c) \in Z(A)$. This concludes the proof.

Corollary 5.2. Given an abstract kernel $\Phi: M \rightarrow \frac{S E n d(A)}{\operatorname{Inn}(A)}$, where $A$ and $M$ are monoids, the group $U(Z(A))$ of $A$ is an $M$-module w.r.t. the action defined by

$$
x \cdot g=\varphi(x)(g) \quad \text { for } x \in M, g \in U(Z(A)), \varphi(x) \in \Phi(x) .
$$

Proof. It is immediate to observe that, if $g \in U(Z(A))$, then $x \cdot g$ is also invertible, with inverse $x \cdot(-g)$, so the action of $M$ on $Z(A)$ restricts to $U(Z(A))$.

Now we describe how to associate an obstruction to an abstract kernel. Given a monoid homomorphism $\Phi: M \rightarrow \frac{\operatorname{SEnd}(A)}{\operatorname{Inn}(A)}$, we choose a representative $\varphi(x) \in \Phi(x)$ for any $x \in M$, with $\varphi(1)=i d_{A}$. We have that

$$
\varphi(x) \varphi(y)=\mu_{f(x, y)} \varphi(x y)
$$

for some $f(x, y) \in U(A)$, with $f(x, 1)=f(1, y)=0$. Now, given $x, y, z \in M$, we have, on one hand

$$
\begin{gathered}
\varphi(x) \varphi(y) \varphi(z)=\varphi(x) \mu_{f(y, z)} \varphi(y z)=\mu_{\varphi(x)(f(y, z))} \varphi(x) \varphi(y z)= \\
=\mu_{\varphi(x)(f(y, z))} \mu_{f(x, y z)} \varphi(x y z)=\mu_{\varphi(x)(f(y, z))+f(x, y z)} \varphi(x y z),
\end{gathered}
$$

and, on the other hand

$\varphi(x) \varphi(y) \varphi(z)=\mu_{f(x, y)} \varphi(x y) \varphi(z)=\mu_{f(x, y)} \mu_{f(x y, z)} \varphi(x y z)=\mu_{f(x, y)+f(x y, z)} \varphi(x y z)$.

Comparing the two expressions, and using the fact that $\varphi(x y z)$ is surjective, we get the equality

$$
\mu_{\varphi(x)(f(y, z))+f(x, y z)}=\mu_{f(x, y)+f(x y, z)},
$$

namely

$$
\mu_{\varphi(x)(f(y, z))+f(x, y z)-(f(x, y)+f(x y, z))}=i d_{A},
$$

which tells us that

$$
\varphi(x)(f(y, z))+f(x, y z)-(f(x, y)+f(x y, z)) \in U(Z(A)) .
$$

This means that there exists a unique element $k(x, y, z) \in U(Z(A))$ such that

$$
\varphi(x)(f(y, z))+f(x, y z)=k(x, y, z)+f(x, y)+f(x y, z) .
$$

Clearly, $k(x, y, 1)=k(x, 1, z)=k(1, y, z)=0$.

Definition 5.3. The function $k: M \times M \times M \rightarrow U(Z(A))$ we get this way is the obstruction of the abstract kernel $\Phi$.

Proposition 5.4. An obstruction $k$ of an abstract kernel $\Phi$ as above is a 3-cocycle of the cohomology of $M$ with coefficients in the $M$-module $U(Z(A))$. 
Proof. Given elements $x, y, z, t \in M$, we compute the expression

$$
\varphi(x)(\varphi(y)(f(z, t))+f(y, z t))+f(x, y z t)
$$

in two different ways. On one hand, we have

$$
\begin{gathered}
\varphi(x)(\varphi(y)(f(z, t))+f(y, z t))+f(x, y z t)=\varphi(x)(k(y, z, t)+f(y, z)+f(y z, t))+f(x, y z t)= \\
=x \cdot k(y, z, t)+\varphi(x)(f(y, z))+\varphi(x)(f(y z, t))+f(x, y z t)= \\
=x \cdot k(y, z, t)+k(x, y, z)+f(x, y)+f(x y, z)-f(x, y z)+ \\
+k(x, y z, t)+f(x, y z)+f(x y z, t)-f(x, y z t)+f(x, y z t)= \\
=x \cdot k(y, z, t)+k(x, y, z)+k(x, y z, t)+f(x, y)+f(x y, z)+f(x y z, t),
\end{gathered}
$$

where the last equality holds since $k$ takes values in the center of $A$. On the other hand, we have

$\varphi(x)(\varphi(y)(f(z, t))+f(y, z t))+f(x, y z t)=\varphi(x) \varphi(y)(f(z, t))+\varphi(x)(f(y, z t))+f(x, y z t)$.

Since $\varphi(x) \varphi(y)=\mu_{f(x, y)} \varphi(x y)$, this is equal to

$$
\begin{gathered}
f(x, y)+\varphi(x y)(f(z, t))-f(x, y)+\varphi(x)(f(y, z t))+f(x, y z t)= \\
=f(x, y)+k(x y, z, t)+f(x y, z)+f(x y z, t)-f(x y, z t)-f(x, y)+k(x, y, z t)+ \\
+f(x, y)+f(x y, z t)-f(x, y z t)+f(x, y z t)= \\
=k(x y, z, t)+k(x, y, z t)+f(x, y)+f(x y, z)+f(x y z, t),
\end{gathered}
$$

where, once again, the last equality holds since $k$ takes values in the center of $A$. Comparing the two expressions, and using the fact that $f$ takes values in $U(A)$, we obtain the equality

$$
x \cdot k(y, z, t)+k(x, y z, t)+k(x, y, z)=k(x y, z, t)+k(x, y, z t) .
$$

Since $k(x, y, 1)=k(x, 1, z)=k(1, y, z)=0$, we have that $k$ is a 3 -cocycle.

In the construction of the obstruction of an abstract kernel $\Phi$, we used the fact that, given $x, y \in M$, there exists an element $f(x, y) \in U(A)$ such that $\varphi(x) \varphi(y)=$ $\mu_{f(x, y)} \varphi(x y)$. Such an element is not unique. However, if we replace it with an $f^{\prime}(x, y)$ with the same properties, the cohomology class of the corresponding 3 cocycle $k^{\prime}$ is the same:

Proposition 5.5. Consider an abstract kernel $\Phi: M \rightarrow \frac{\operatorname{SEnd}(A)}{\operatorname{Inn}(A)}$, with chosen representatives $\varphi(x) \in \Phi(x)$ for any $x \in M$, with $\varphi(1)=i d_{A}$. If, for any $x, y \in M$, we have

with

$$
\varphi(x) \varphi(y)=\mu_{f(x, y)} \varphi(x y)=\mu_{f^{\prime}(x, y)} \varphi(x y)
$$

$$
f(x, 1)=0=f(1, y) \quad \text { and } \quad f^{\prime}(x, 1)=0=f^{\prime}(1, y)
$$

then the 3 -cocycles $k$ and $k^{\prime}$ constructed using $f$ and $f^{\prime}$ are cohomologous.

Proof. From the equality

$$
\mu_{f(x, y)} \varphi(x y)=\mu_{f^{\prime}(x, y)} \varphi(x y)
$$

we get $\mu_{f(x, y)}=\mu_{f^{\prime}(x, y)}$, because $\varphi(x y)$ is surjective. This means that

$$
\mu_{f(x, y)-f^{\prime}(x, y)}=i d_{A} .
$$

Hence

$$
h(x, y)=f(x, y)-f^{\prime}(x, y) \in U(Z(A)),
$$

so we get a map

$$
h: M \times M \rightarrow U(Z(A))
$$

such that $h(x, 1)=0=h(1, y)$. From the equality

$$
f(x, y)=h(x, y)+f^{\prime}(x, y),
$$


valid for all $x, y \in M$, and from the definition of the cocycles $k$ and $k^{\prime}$, we get:

$$
\begin{gathered}
k(x, y, z)=\varphi(x)(f(y, z))+f(x, y z)-f(x y, z)-f(x, y)= \\
=\varphi(x)\left(h(y, z)+f^{\prime}(y, z)\right)+h(x, y z)+f^{\prime}(x, y z)-\left[h(x y, z)+f^{\prime}(x y, z)\right]-\left[h(x, y)+f^{\prime}(x, y)\right]= \\
=\varphi(x)\left(f^{\prime}(y, z)\right)+f^{\prime}(x, y z)-f^{\prime}(x y, z)-f^{\prime}(x, y)+x \cdot h(y, z)-h(x y, z)+h(x, y z)-h(x, y)= \\
=k^{\prime}(x, y, z)-\delta^{2} h(x, y, z) .
\end{gathered}
$$

Thus $k^{\prime}-k=\delta^{2} h$.

Conversely, starting with cohomologous cocycles:

Proposition 5.6. Consider an abstract kernel $\Phi: M \rightarrow \frac{S E n d(A)}{\operatorname{Inn}(A)}$, with chosen representatives $\varphi(x) \in \Phi(x)$ for any $x \in M$, with $\varphi(1)=i d_{A}$. Let $f: M \times M \rightarrow U(A)$ be a map with $\varphi(x) \varphi(y)=\mu_{f(x, y)} \varphi(x y)$ and $f(x, 1)=0=f(1, y)$ for any $x, y \in M$, and let $k: M \times M \times M \rightarrow U(Z(A))$ be the 3-cocycle induced by $f$. If $k^{\prime \prime}$ is a 3cocycle which is cohomologous to $k$, then there exists a map $f^{\prime \prime}: M \times M \rightarrow U(A)$, with $f^{\prime \prime}(x, 1)=0=f^{\prime \prime}(1, y)$, such that

$$
\varphi(x) \varphi(y)=\mu_{f^{\prime \prime}(x, y)} \varphi(x y)
$$

and the 3 -cocycle induced by $f^{\prime \prime}$ is precisely $k^{\prime \prime}$.

Proof. By assumption, there exists a map $h: M \times M \rightarrow U(Z(A))$, with $h(x, 1)=$ $0=h(1, y)$, such that $k-k^{\prime \prime}=\delta^{2} h$. We define $f^{\prime \prime}: M \times M \rightarrow U(A)$ by putting

$$
f^{\prime \prime}(x, y)=h(x, y)+f(x, y) \text {. }
$$

Clearly $f^{\prime \prime}(x, 1)=0=f^{\prime \prime}(1, y)$. Moreover,

$$
\mu_{f^{\prime \prime}(x, y)}=\mu_{h(x, y)} \mu_{f(x, y)}=\mu_{f(x, y)}
$$

since $h(x, y) \in U(Z(A))$. Therefore

$$
\varphi(x) \varphi(y)=\mu_{f^{\prime \prime}(x, y)} \varphi(x y) .
$$

Furthermore, for any $x, y, z \in M$ we have

$$
\begin{gathered}
\varphi(x)\left(f^{\prime \prime}(y, z)\right)+f^{\prime \prime}(x, y z)-f^{\prime \prime}(x y, z)-f^{\prime \prime}(x, y)= \\
=\varphi(x)(h(y, z)+f(y, z))+h(x, y z)+f(x, y z)-[h(x y, z)+f(x y, z)]-[h(x, y)+f(x, y)]= \\
=\varphi(x)(f(y, z))+f(x, y z)-f(x y, z)-f(x, y)+x \cdot h(y, z)-h(x y, z)+h(x, y z)-h(x, y)= \\
=k(x, y, z)-\delta^{2} h(x, y, z)=k^{\prime \prime}(x, y, z) .
\end{gathered}
$$

It remains to check what happens if, given an abstract kernel $\Phi: M \rightarrow \frac{\operatorname{SEnd}(A)}{\operatorname{Inn}(A)}$, we consider two different representatives $\varphi(x)$ and $\varphi^{\prime}(x)$ of $\Phi(x)$ :

Proposition 5.7. Consider an abstract kernel $\Phi: M \rightarrow \frac{\operatorname{SEnd}(A)}{\operatorname{Inn}(A)}$, with chosen representatives $\varphi(x) \in \Phi(x)$ for any $x \in M$, with $\varphi(1)=i d_{A}$. Let $f: M \times M \rightarrow U(A)$ be a map with $\varphi(x) \varphi(y)=\mu_{f(x, y)} \varphi(x y)$ and $f(x, 1)=0=$ $f(1, y)$ for any $x, y \in M$, and let $k: M \times M \times M \rightarrow U(Z(A))$ be the 3 -cocycle induced by $f$. If one chooses other representatives $\varphi^{\prime}(x) \in \Phi(x)$, again with $\varphi^{\prime}(1)=i d_{A}$, then there exists a map $f^{\prime}: M \times M \rightarrow U(A)$, with $f^{\prime}(x, 1)=0=f^{\prime}(1, y)$, such that

$$
\varphi^{\prime}(x) \varphi^{\prime}(y)=\mu_{f^{\prime}(x, y)} \varphi^{\prime}(x y)
$$

and its induced 3-cocycle is precisely $k$. 
Proof. Since $\varphi(x), \varphi^{\prime}(x) \in \Phi(x)$ for all $x \in M$, they differ by an inner automorphism of $A$. In other terms, there is a map $g: M \rightarrow U(A)$, with $g(1)=0$, such that

$$
\varphi^{\prime}(x)=\mu_{g(x)} \varphi(x) \text {. }
$$

Then, for $x, y \in M$, we get

$$
\begin{gathered}
\varphi^{\prime}(x) \varphi^{\prime}(y)=\mu_{g(x)} \varphi(x) \mu_{g(y)} \varphi(y)=\mu_{g(x)} \mu_{\varphi(x)(g(y))} \varphi(x) \varphi(y)= \\
=\mu_{g(x)} \mu_{\varphi(x)(g(y))} \mu_{f(x, y)} \varphi(x y)=\mu_{g(x)} \mu_{\varphi(x)(g(y))} \mu_{f(x, y)} \mu_{g(x y)}^{-1} \varphi^{\prime}(x y)= \\
=\mu_{g(x)} \mu_{\varphi(x)(g(y))} \mu_{f(x, y)} \mu_{-g(x y)} \varphi^{\prime}(x y)=\mu_{g(x)+\varphi(x)(g(y))+f(x, y)-g(x y)} \varphi^{\prime}(x y) .
\end{gathered}
$$

Thus, defining

$$
f^{\prime}(x, y)=g(x)+\varphi(x)(g(y))+f(x, y)-g(x y)
$$

we obtain that $\varphi^{\prime}(x) \varphi^{\prime}(y)=\mu_{f^{\prime}(x, y)} \varphi^{\prime}(x y)$, and obviously $f^{\prime}(x, 1)=0=f^{\prime}(1, y)$. It remains to check that the induced 3-cocycle is $k$. We have

$$
\begin{gathered}
\varphi^{\prime}(x)\left(f^{\prime}(y, z)\right)+f^{\prime}(x, y z)-f^{\prime}(x y, z)-f^{\prime}(x, y)= \\
=\varphi^{\prime}(x)[g(y)+\varphi(y)(g(z))+f(y, z)-g(y z)]+g(x)+\varphi(x)(g(y z))+f(x, y z)-g(x y z)+ \\
-[g(x y)+\varphi(x y)(g(z))+f(x y, z)-g(x y z)]-[g(x)+\varphi(x)(g(y))+f(x, y)-g(x y)]= \\
=\mu_{g(x)}[\varphi(x)(g(y))+\varphi(x) \varphi(y)(g(z))+\varphi(x)(f(y, z))-\varphi(x)(g(y z))]+g(x)+\varphi(x)(g(y z))+ \\
+f(x, y z)-g(x y z)+g(x y z)-f(x y, z)-\varphi(x y)(g(z))-g(x y)+g(x y)-f(x, y)-\varphi(x)(g(y))-g(x)= \\
=g(x)+\varphi(x)(g(y))+\varphi(x) \varphi(y)(g(z))+\varphi(x)(f(y, z))-\varphi(x)(g(y z))-g(x)+g(x)+ \\
+\varphi(x)(g(y z))+f(x, y z)-f(x y, z)-\varphi(x y)(g(z))-f(x, y)-\varphi(x)(g(y))-g(x)= \\
=g(x)+\varphi(x)(g(y))+\varphi(x) \varphi(y)(g(z))+\varphi(x)(f(y, z))+f(x, y z)-f(x y, z)+ \\
\quad-\varphi(x y)(g(z))-f(x, y)-\varphi(x)(g(y))-g(x)= \\
=g(x)+\varphi(x)(g(y))+\mu f(x, y) \varphi(x y)(g(z))+k(x, y, z)+f(x, y)-\varphi(x y)(g(z))-f(x, y)+ \\
\quad-\varphi(x)(g(y))-g(x)= \\
=k(x, y, z)+g(x)+\varphi(x)(g(y))+f(x, y)+\varphi(x y)(g(z))-f(x, y)+f(x, y)+ \\
\quad-\varphi(x y)(g(z))-f(x, y)-\varphi(x)(g(y))-g(x)= \\
\quad=k(x, y, z),
\end{gathered}
$$

and this concludes the proof.

The previous propositions give the following:

Theorem 5.8. Any abstract kernel $\Phi: M \rightarrow \frac{\operatorname{SEnd}(A)}{\operatorname{Inn}(A)}$ determines in an invariant way an element $\operatorname{Obs}(\Phi)$ of the third cohomology group $H^{3}(M, U(Z(A)))$ of the monoid $M$ with coefficients in the $M$-module $U(Z(A))$. An abstract kernel $\Phi: M \rightarrow \frac{S E n d(A)}{\operatorname{Inn}(A)}$ is induced by a regular weakly homogeneous Schreier extension if and only if $\mathrm{Obs}(\Phi)$ is the zero element of $H^{3}(M, U(Z(A)))$.

Proof. The fact that the element $\operatorname{Obs}(\Phi) \in H^{3}(M, U(Z(A)))$ is uniquely determined is a consequence of the previous propositions. If the abstract kernel $\Phi$ is induced by a regular weakly homogeneous Schreier extension, we observed at the beginning of Section 4 that there exists a map $f: M \times M \rightarrow U(A)$ such that $f(x, 1)=0=f(1, y)$ and

$$
\varphi(x)(f(y, z))+f(x, y z)=f(x, y)+f(x y, z) \quad \text { for all } x, y, z \in M .
$$

Hence, the element $\operatorname{Obs}(\Phi)$ associated to the abstract kernel $\Phi$ induced by the extension is zero. Conversely, if the obstruction of an abstract kernel $\Phi: M \rightarrow \frac{\operatorname{SEnd}(A)}{\operatorname{Inn}(A)}$ is zero, then the crossed product extension built in Proposition 4.1 (which is weakly homogeneous by Remark 4.2) induces $\Phi$. 
In particular, for abstract kernels which factor through $\frac{A u t(A)}{\operatorname{Inn}(A)}$, i.e. for abstract kernels of the form $\Phi: M \rightarrow \frac{A u t(A)}{\operatorname{Inn}(A)}$, we get:

Theorem 5.9. Any abstract kernel $\Phi: M \rightarrow \frac{A u t(A)}{\operatorname{Inn}(A)}$ determines in an invariant way an element $\operatorname{Obs}(\Phi)$ of the third cohomology group $H^{3}(M, U(Z(A)))$ of the monoid $M$ with coefficients in the $M$-module $U(Z(A))$. An abstract kernel $\Phi: M \rightarrow \frac{A u t(A)}{\operatorname{Inn}(A)}$ is induced by a regular homogeneous Schreier extension if and only if $\operatorname{Obs}(\Phi)$ is the zero element of $H^{3}(M, U(Z(A)))$.

If the monoid $A$ is a group, then $S \operatorname{End}(A)=\operatorname{Epi}(A)$ and every Schreier extension of $M$ by $A$ is regular (see Proposition 3.10). Recalling that such extensions are called special Schreier in $[4,5,17,18]$, it is worth mentioning the following particular case of the previous theorems:

Corollary 5.10. Let $M$ be a monoid and $A$ a group. Any abstract kernel $\Phi: M \rightarrow \frac{\operatorname{Epi}(A)}{\operatorname{Inn}(A)}$ (resp. $\left.\Phi: M \rightarrow \frac{\operatorname{Aut}(A)}{\operatorname{Inn}(A)}\right)$ determines in an invariant way an element $\operatorname{Obs}(\Phi)$ of the third cohomology group $H^{3}(M, Z(A))$ of the monoid $M$ with coefficients in the $M$-module $Z(A)$. An abstract kernel $\Phi: M \rightarrow \frac{\operatorname{Epi}(A)}{\operatorname{Inn}(A)}$ (resp. $\left.\Phi: M \rightarrow \frac{A u t(A)}{\operatorname{Inn}(A)}\right)$ is induced by a weakly homogeneous (resp. homogeneous) special Schreier extension if and only if $\mathrm{Obs}(\Phi)$ is the zero element of $H^{3}(M, Z(A))$.

We observe that the particular case described in the previous corollary could also be obtained from the results of [27].

\section{The Classification of REgular WeAkly homogeneous AND REgular HOMOGENEOUS SCHREIER EXTENSIONS}

In this section we show that the set $\operatorname{Ext}(M, A, \Phi)$ of isomorphism classes of regular weakly homogeneous (resp. homogeneous) Schreier extensions (1) which induce the same abstract kernel $\Phi: M \rightarrow \frac{\operatorname{SEnd}(A)}{\operatorname{Inn}(A)}$ (resp. $\left.\Phi: M \rightarrow \frac{\operatorname{Aut}(A)}{\operatorname{Inn}(A)}\right)$, when it is not empty, is in bijection with the second cohomology group $H^{2}(M, U(Z(A)))$ of $M$ with coefficients in the $M$-module $U(Z(A))$. In order to do this, we show that there is a simply transitive action of the abelian group $H^{2}(M, U(Z(A)))$ on the set $\operatorname{Ext}(M, A, \Phi)$.

We start by recalling that an action of a group $G$ on a set $S$ is simply transitive if, for all $s, s^{\prime} \in S$, there exists a unique $g \in G$ such that $g \cdot s=s^{\prime}$. Given a simply transitive action of $G$ on $S$, every element $s \in S$ determines then a bijection $\alpha: G \rightarrow S$, defined by $\alpha(g)=g \cdot s$.

Suppose now that an abstract kernel $\Phi: M \rightarrow \frac{\operatorname{SEnd}(A)}{\operatorname{Inn}(A)}$ is induced by a regular weakly homogeneous Schreier extension (1), i.e. that the set $\operatorname{Ext}(M, A, \Phi)$ is not empty. For every $x \in M$, we choose a representative $\varphi(x) \in \Phi(x)$, with $\varphi(1)=i d_{A}$. We define an action of $H^{2}(M, U(Z(A)))$ on $\operatorname{Ext}(M, A, \Phi)$ as follows. Given elements $c l(h) \in H^{2}(M, U(Z(A)))$ and $\operatorname{cl}(E) \in \operatorname{Ext}(M, A, \Phi)$, Proposition 4.4 tells us that $E$ is isomorphic to a crossed product extension $[A, \varphi, f, M]$, where $f: M \times M \rightarrow U(A)$ is a map with $f(x, 1)=0=f(1, y)$ and

$$
\varphi(x) \varphi(y)=\mu_{f(x, y)} \varphi(x y)
$$

and such that the equality (3) holds. Consider the function $h+f: M \times M \rightarrow U(A)$ defined by $(h+f)(x, y)=h(x, y)+f(x, y)$. Clearly

$$
(h+f)(x, 1)=0=(h+f)(1, y),
$$


and, since $h(x, y) \in U(Z(A))$, we also have

$$
\varphi(x) \varphi(y)=\mu_{(h+f)(x, y)} \varphi(x y) .
$$

Furthermore,

$$
\begin{gathered}
\varphi(x)(h(y, z)+f(y, z))+h(x, y z)+f(x, y z)= \\
=\varphi(x)(h(y, z))+\varphi(x)(f(y, z))+h(x, y z)+f(x, y z)= \\
=\varphi(x)(h(y, z))+h(x, y z)+\varphi(x)(f(y, z))+f(x, y z)= \\
=h(x, y)+h(x y, z)+f(x, y)+f(x y, z)= \\
=h(x, y)+f(x, y)+h(x y, z)+f(x y, z),
\end{gathered}
$$

where we are using that $h(x, y z), h(x y, z) \in U(Z(A))$, that $h$ is a 2-cocycle and the equality (3). Thus

$$
\varphi(x)(h+f)(y, z)+(h+f)(x, y z)=(h+f)(x, y)+(h+f)(x y, z) .
$$

Thanks to this equality, we can build the crossed product extension $[A, \varphi, h+f, M]$, which is weakly homogeneous by Remark 4.2. The action of $H^{2}(M, U(Z(A)))$ on $\operatorname{Ext}(M, A, \Phi)$ we are looking for is then defined by:

$$
\operatorname{cl}(h) \cdot \operatorname{cl}(E)=\operatorname{cl}([A, \varphi, h+f, M]) .
$$

Theorem 6.1. The action (4) is well defined and simply transitive.

Proof. We first prove that the action is well defined, i.e. that it does not depend on the choice of the representatives. If $\operatorname{cl}(h)=\operatorname{cl}\left(h^{\prime}\right)$ and $\operatorname{cl}(E)=\operatorname{cl}\left(E^{\prime}\right)$, with $E^{\prime}$ isomorphic to the crossed product extension $\left[A, \varphi, f^{\prime}, M\right]$, then

$$
\operatorname{cl}\left(h^{\prime}\right) \cdot \operatorname{cl}\left(E^{\prime}\right)=\operatorname{cl}\left(\left[A, \varphi, h^{\prime}+f^{\prime}, M\right]\right) .
$$

Since $c l(E)=\operatorname{cl}\left(E^{\prime}\right)$, there exists a commutative diagram

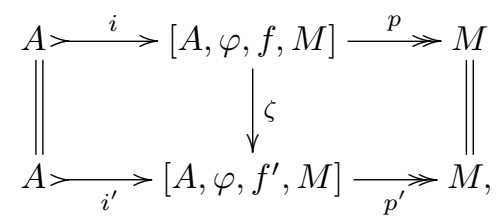

where $\zeta$ is an isomorphism. For every $x \in M$ we have $\zeta(0, x)=(r(x), x)$ with $r(x) \in U(A)$. Indeed, $\zeta$ sends representatives to representatives, and $(a, x)$ is, by Proposition 4.1, a representative if and only if $a \in U(A)$. Moreover

$$
(r(1), 1)=\zeta(0,1)=(0,1),
$$

whence $r(1)=0$. So, we get a map $r: M \rightarrow U(A)$ with $r(1)=0$. Furthermore, for all $a \in A$ and $x \in M$ we have

$$
\begin{gathered}
\zeta(a, x)=\zeta((a, 1)+(0, x))=\zeta(a, 1)+\zeta(0, x)=(a, 1)+(r(x), x)= \\
=\left(a+\varphi(1) r(x)+f^{\prime}(1, x), x\right)=(a+r(x), x) .
\end{gathered}
$$

Using this equality, one gets

$\zeta\left(\left(a_{1}, x\right)+\left(a_{2}, y\right)\right)=\zeta\left(a_{1}+\varphi(x)\left(a_{2}\right)+f(x, y), x y\right)=\left(a_{1}+\varphi(x)\left(a_{2}\right)+f(x, y)+r(x y), x y\right)$ and

$$
\begin{gathered}
\zeta\left(\left(a_{1}, x\right)+\left(a_{2}, y\right)\right)=\zeta\left(a_{1}, x\right)+\zeta\left(a_{2}, y\right)=\left(a_{1}+r(x), x\right)+\left(a_{2}+r(y), y\right)= \\
=\left(a_{1}+r(x)+\varphi(x)\left(a_{2}\right)+\varphi(x)(r(y))+f^{\prime}(x, y), x y\right) .
\end{gathered}
$$

Comparing the two expressions, we obtain

(5) $a_{1}+\varphi(x)\left(a_{2}\right)+f(x, y)+r(x y)=a_{1}+r(x)+\varphi(x)\left(a_{2}\right)+\varphi(x)(r(y))+f^{\prime}(x, y)$.

Moreover, since $\operatorname{cl}(h)=\operatorname{cl}\left(h^{\prime}\right)$, there is a 1-cochain $\gamma: M \rightarrow U(Z(A))$ such that

$$
h(x, y)+\gamma(x y)=h^{\prime}(x, y)+x \cdot \gamma(y)+\gamma(x) .
$$


Consider now the diagram

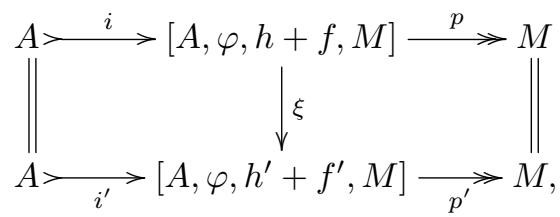

where the map $\xi$ is defined by $\xi(a, x)=(\gamma(x)+a+r(x), x)$. Clearly the diagram is commutative. Using the equalities (5) and (6), and the fact that $h, h^{\prime}$ and $\gamma$ take values in $U(Z(A))$, it is straightforward to check that $\xi$ is a monoid homomorphism. Moreover, it sends representatives to representatives, since $\xi(a, x)=(\gamma(x)+a+$ $r(x), x)$, and $\gamma(x)+a+r(x) \in U(A)$ whenever $a \in U(A)$ (see Proposition 4.1). Then, Proposition 4.3 implies that $\xi$ is an isomorphism. This shows that the action is well defined. It is obviously an action, since

$$
\left(\operatorname{cl}(h)+\operatorname{cl}\left(h^{\prime}\right)\right) \cdot \operatorname{cl}(E)=\operatorname{cl}(h) \cdot\left(\operatorname{cl}\left(h^{\prime}\right) \cdot \operatorname{cl}(E)\right) \quad \text { and } \quad c l(0) \cdot \operatorname{cl}(E)=\operatorname{cl}(E) .
$$

The next step of the proof consists in showing that the action is simple, namely:

$$
\operatorname{cl}\left(h_{1}\right) \cdot \operatorname{cl}(E)=\operatorname{cl}\left(h_{2}\right) \cdot \operatorname{cl}(E) \quad \Rightarrow \quad \operatorname{cl}\left(h_{1}\right)=\operatorname{cl}\left(h_{2}\right) .
$$

If $c l\left(h_{1}\right) \cdot \operatorname{cl}(E)=\operatorname{cl}\left(h_{2}\right) \cdot \operatorname{cl}(E)$, we have a commutative diagram

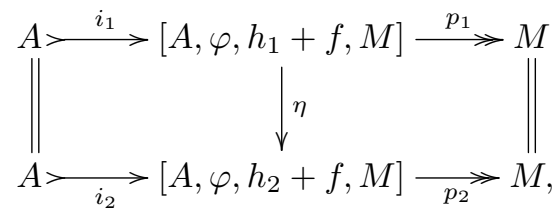

where $\eta$ is an isomorphism. As we did for $\zeta$ in the first part of the proof, one can check that

$$
\eta(a, x)=(a+b(x), x), \quad \text { with } b: M \rightarrow U(A), \quad b(1)=0 .
$$

Let us prove that $b$ is in fact a 1-cochain with $h_{1}-h_{2}=\delta^{1} b$. If $a \in A$ and $x \in M$, then there exists $a^{\prime} \in A$ such that $\varphi(x)\left(a^{\prime}\right)=a$, because $\varphi(x) \in \operatorname{SEnd}(A)$. Then we get

$$
\begin{gathered}
(a+b(x), x)=\eta(a, x)=\eta\left(\varphi(x)\left(a^{\prime}\right), x\right)=\eta\left((0, x)+\left(a^{\prime}, 1\right)\right)=\eta(0, x)+\eta\left(a^{\prime}, 1\right)= \\
=(b(x), x)+\left(a^{\prime}, 1\right)=\left(b(x)+\varphi(x)\left(a^{\prime}\right), x\right)=(b(x)+a, x),
\end{gathered}
$$

hence $a+b(x)=b(x)+a$, which means that $b(x) \in U(Z(A))$ for all $x \in M$. Moreover

$$
\eta((0, x)+(0, y))=\eta\left(h_{1}(x, y)+f(x, y), x y\right)=\left(h_{1}(x, y)+f(x, y)+b(x y), x y\right)
$$

and

Therefore

$$
\begin{gathered}
\eta((0, x)+(0, y))=\eta(0, x)+\eta(0, y)=(b(x), x)+(b(y), y)= \\
=\left(b(x)+\varphi(x)(b(y))+h_{2}(x, y)+f(x, y), x y\right) .
\end{gathered}
$$

$$
h_{1}(x, y)+f(x, y)+b(x y)=b(x)+\varphi(x)(b(y))+h_{2}(x, y)+f(x, y),
$$

whence

$$
h_{1}(x, y)-h_{2}(x, y)=\varphi(x)(b(y))-b(x y)+b(x)=\delta^{1} b(x, y)
$$

and this tells us that $\operatorname{cl}\left(h_{1}\right)=\operatorname{cl}\left(h_{2}\right)$ and the action is simple.

It remains to prove that it is transitive, i.e. that for all $\operatorname{cl}(E), \operatorname{cl}\left(E^{\prime}\right) \in \operatorname{Ext}(M, A, \Phi)$ there exists $c l(h) \in H^{2}(M, U(Z(A)))$ such that $c l(h) \cdot \operatorname{cl}(E)=\operatorname{cl}\left(E^{\prime}\right)$. Given $\operatorname{cl}(E), \operatorname{cl}\left(E^{\prime}\right) \in \operatorname{Ext}(M, A, \Phi)$, we know from Proposition 4.4 that $E$ and $E^{\prime}$ are 
isomorphic to crossed product extensions $[A, \varphi, f, M]$ and $\left[A, \varphi, f^{\prime}, M\right]$ respectively, where for all $x, y \in M$ the following equalities hold:

$$
\varphi(x) \varphi(y)=\mu_{f(x, y)} \varphi(x y)=\mu_{f^{\prime}(x, y)} \varphi(x y) .
$$

Being $\varphi(x y)$ surjective, this implies that $\mu_{f^{\prime}(x, y)-f(x, y)}=i d_{A}$, and so $f^{\prime}(x, y)-$ $f(x, y) \in U(Z(A))$. Let us then define the function $h: M \times M \rightarrow U(Z(A))$ by putting

$$
h(x, y)=f^{\prime}(x, y)-f(x, y) .
$$

A straightforward calculation (using the equality (3) and the fact that $h$ takes values in $U(Z(A)))$ shows that $h$ is a 2 -cocycle. Then we get

$$
\operatorname{cl}(h) \cdot \operatorname{cl}(E)=\operatorname{cl}([A, \varphi, h+f, M])=\operatorname{cl}\left(\left[A, \varphi, f^{\prime}, M\right]\right)=\operatorname{cl}\left(E^{\prime}\right),
$$

and the action is transitive.

The previous theorem gives then the desired bijection between $\operatorname{Ext}(M, A, \Phi)$ and $H^{2}(M, U(Z(A)))$ :

Corollary 6.2. For any fixed $\operatorname{cl}(E) \in \operatorname{Ext}(M, A, \Phi)$, the map from $H^{2}(M, U(Z(A)))$ to $\operatorname{Ext}(M, A, \Phi)$ which sends $\operatorname{cl}(h)$ to $\operatorname{cl}(h) \cdot \operatorname{cl}(E)$ is bijective.

If $U(Z(A))=0$ (in particular, if $U(A)=0$ or $Z(A)=0$ ), then both $H^{2}(M, U(Z(A)))$ and $H^{3}(M, U(Z(A)))$ are the trivial groups. This means that, for every abstract kernel $\Phi: M \rightarrow \frac{\operatorname{SEnd}(A)}{\operatorname{Inn}(A)}, \operatorname{Obs}(\Phi)=0$. Hence we get the following

Corollary 6.3. If $U(Z(A))=0$, for every abstract kernel $\Phi: M \rightarrow \frac{\operatorname{SEnd}(A)}{\operatorname{Inn}(A)}$, there exists, up to isomorphism, a unique weakly homogeneous Schreier extension of $M$ by $A$ which induces $\Phi$. If $U(A)=0$, then also $\operatorname{Inn}(A)=0$, and so the abstract kernel is a monoid homomorphism $\Phi: M \rightarrow S E n d(A)$, i.e. an action of $M$ on $A$. In this case the unique weakly homogeneous extension is the semidirect product of $M$ and $A$ via the action $\Phi$.

It is immediate to see that the results of this section are valid, in particular, for abstract kernels of the form $\Phi: M \rightarrow \frac{\operatorname{Aut}(A)}{\operatorname{Inn}(A)}$ and regular homogeneous Schreier extensions. Let us state them explicitly.

Theorem 6.4. Given an abstract kernel $\Phi: M \rightarrow \frac{A u t(A)}{\operatorname{Inn}(A)}$, if the set $\operatorname{Ext}(M, A, \Phi)$ of isomorphism classes of regular homogeneous Schreier extensions of $M$ by $A$ which induce $\Phi$ is not empty, then (4) is a simply transitive action of the abelian group $H^{2}(M, U(Z(A)))$ on $\operatorname{Ext}(M, A, \Phi)$. This action induces a bijection between $\operatorname{Ext}(M, A, \Phi)$ and $H^{2}(M, U(Z(A)))$.

Corollary 6.5. If the monoid $A$ is such that $U(Z(A))=0$, for every abstract kernel $\Phi: M \rightarrow \frac{A u t(A)}{\operatorname{Inn}(A)}$ there exists, up to isomorphism, a unique homogeneous Schreier extension of $M$ by $A$ which induces $\Phi$. If $U(A)=0$, then also $\operatorname{Inn}(A)=0$, and so the abstract kernel is a monoid homomorphism $\Phi: M \rightarrow A u t(A)$, i.e. an action of $M$ on $A$. In this case the unique homogeneous extension is the semidirect product of $M$ and $A$ via the action $\Phi$.

Finally note that, if $A$ and $M$ are both groups, then Theorem 6.4 turns into the classical cohomological classification of group extensions with non-abelian kernel (see, e.g., [16]). 


\section{ACKNOWLEDGEMENTS}

This work was partially supported by the Centre for Mathematics of the University of Coimbra - UID/MAT/00324/2019, by ESTG and CDRSP from the Polytechnical Institute of Leiria - UID/Multi/04044/2019, funded by the Portuguese Government through FCT/MCTES and co-funded by the European Regional Development Fund through the Partnership Agreement PT2020.

The second author was partially supported by the Programma per Giovani Ricercatori "Rita Levi-Montalcini", funded by the Italian government through MIUR.

The third author was supported by the Shota Rustaveli National Science Foundation of Georgia (SRNSFG), grant FR-18-10849, "Stable Structures in Homological Algebra".

\section{REFERENCES}

[1] D. Bourn, Commutator theory, action groupoids, and an intrinsic Schreier-Mac Lane extension theorem, Adv. in Math. 217 (2008), 2700-2735.

[2] D. Bourn, Internal profunctors and commutator theory; applications to extensions classification and categorical Galois theory, Theory Appl. Categ. 24 (2010), 451-488.

[3] D. Bourn, G. Janelidze, Centralizers in action accessible categories, Cahiers Top. Géom. Différ. Catég. 50 (2009), no. 3, 211-232.

[4] D. Bourn, N. Martins-Ferreira, A. Montoli, M. Sobral, Schreier split epimorphisms in monoids and in semirings, Textos de Matemática (Série B), Departamento de Matemática da Universidade de Coimbra, vol. 45 (2013).

[5] D. Bourn, N. Martins-Ferreira, A. Montoli, M. Sobral, Schreier split epimorphisms between monoids, Semigroup Forum 88 (2014), 739-752.

[6] D. Bourn, A. Montoli, Intrinsic Schreier-Mac Lane extension theorem II: the case of action accessible categories, J. Pure Appl. Algebra 216 (2012), 1757-1767.

[7] P. Carrasco, A.R. Garzón, Obstruction theory for extensions of categorical groups, Appl. Categ. Structures 12 (2004), 35-61.

[8] A.S. Cigoli, G. Metere, Extension theory and the calculus of butterflies, J. Algebra 458 (2016), $87-119$.

[9] A.S. Cigoli, G. Metere, A. Montoli, Obstruction theory in action accessible categories, J. Algebra 385 (2013), 27-46.

[10] S. Eilenberg, S. Mac Lane, Cohomology theory in abstract groups II. Group extensions with a non-abelian kernel, Ann. of Math. (2) 48 (1947), 326-341.

[11] A.R. Garzón, H. Inassaridze, Semidirect products of categorical groups. Obstruction theory, Homol. Homot. Appl. 3 (2001), 111-138.

[12] G. Hochschild, Cohomology and representation of associative algebras, Duke Math. J. 14 (1947), 921-948.

[13] G. Hochschild, Lie algebra kernels and cohomology, Amer. J. Math. 76 (1954), 698-716.

[14] G. Janelidze, L. Márki, W. Tholen, Semi-abelian categories, J. Pure Appl. Algebra 168 (2002), $367-386$.

[15] S. Mac Lane, Extensions and obstructions for rings, Illinois J. Math. 2 (1958), 316-345.

[16] S. Mac Lane, Homology, Springer-Verlag, 1963.

[17] N. Martins-Ferreira, A. Montoli, M. Sobral, Baer sums of special Schreier extensions of monoids, Semigroup Forum 93 (2016), 403-415.

[18] N. Martins-Ferreira, A. Montoli, M. Sobral, The Nine Lemma and the push forward construction for special Schreier extensions of monoids with operations, Semigroup Forum 97 (2018), 325-352.

[19] G. Orzech, Obstruction theory in algebraic categories, I, J. Pure Appl. Algebra 2 (1972), 287-314.

[20] A. Patchkoria, Extensions of semimodules by monoids and their cohomological characterization, Bull. Georgian Acad. Sci. 86 (1977), 21-24 (in Russian).

[21] A. Patchkoria, Cohomology of monoids with coefficients in semimodules, Bull. Georgian Acad. Sci. 86 (1977), 545-548 (in Russian).

[22] A. Patchkoria, On Schreier extensions of semimodules, PhD thesis, Tbilisi State University, 1979 (in Russian).

[23] A. Patchkoria, Cohomology monoids of monoids with coefficients in semimodules II, Semigroup Forum 97 (2018), 131-153. 
[24] L. Rédei, Die Verallgemeinerung der Schreierischen Erweiterungstheorie, Acta Sci. Math. Szeged 14 (1952), 252-273.

[25] O. Schreier, Über die Erweiterung von Gruppen I, Monathsh. Math. 34 (1926), 165-180.

[26] O. Schreier, Über die Erweiterung von Gruppen II, Abh. Math. Sem. Univ. Hamburg 4 (1926), 276-280.

[27] N. S. Tuen, Non-abelian extensions of monoids, Bull. Georgian Acad. Sci. 84 (1976), 37-39 (in Russian).

(Nelson Martins-Ferreira) ESTG, CDRSP, Instituto Politécnico de Leiria, Leiria, PorTUGAL

Email address: martins.ferreira@ipleiria.pt

(Andrea Montoli) Dipartimento di Matematica "Federigo Enriques", Università Degli Studi di Milano, Via Saldini 50, 20133 Milano, Italy

Email address: andrea.montoli@unimi.it

(Alex Patchkoria) A.Razmadze Mathematical Institute, Ivane Javakhishvili Tbilisi State University, Tamarashvili Str. 6, Tbilisi 0177, Georgia

Email address: alex.patchkoria@googlemail.com

(Manuela Sobral) CMUC and Departamento de Matemática, Universidade de Coimbra, 3001-501 Coimbra, Portugal

Email address: sobral@mat.uc.pt 